\title{
Structural optimization in ESAC: annals 2011
}

\author{
Ji-Hong Zhu* and Wei-Hong Zhang \\ Engineering Simulation and Aerospace Computing, Northwestern Polytechnical University, 710072, Xi'an, Shaanxi, P.R. China
}

Received 2 June 2012 / Accepted 23 October 2013 / Published online 6 February 2014

\begin{abstract}
The purpose of this paper is to give an overall introduction of the structural optimization research works in ESAC group in 2011. Four main topics are involved, i.e. 1) topology optimization with multiphase materials, 2) integrated layout and topology optimization, 3) prediction of effective material properties and 4) composite design. More detailed techniques and some numerical results are also presented and discussed here.
\end{abstract}

Key words: ESAC annals, Topology optimization, Shape optimization, Composite.

\section{Introduction}

The Laboratory of Engineering Simulation and Aerospace Computing (ESAC) in Northwestern Polytechnical University is an active international research team in the field of structural optimization design. Since 1990s, we have been dedicated to the theoretical study of shape and topology optimization and their applications in aeronautics and aerospace industries.

In 2011, the research works and the published papers are mainly concentrated on the research of the following four topics, i.e., (1) topology optimization with multiphase materials, (2) integrated layout and topology optimization, (3) prediction of effective material properties and (4) composite design.

\section{Topology optimization with multiphase materials}

This work is focused on the topology optimization of lightweight structures consisting of multiphase materials $[1,2]$. Instead of adopting the common idea of using volume constraint, a new problem formulation with mass constraint is firstly proposed, which is a significant breakthrough of the topology optimization with multiphase materials. For a discretized structure with $n$ designable finite elements, the mass constraint is stated as

$$
\sum_{i=1}^{n} \rho_{i} V_{i} \leq M,
$$

where $\rho_{i}$ denotes the material density related to element $i$, which has to be interpolated from the set of available material

\footnotetext{
*e-mail: jh.zhu@nwpu.edu.cn
}

densities. $M$ is the upper bound controlling the structural mass.

Meanwhile, to have a deep understanding of the mass constraint, recursive multiphase materials interpolation (RMMI) and uniform multiphase materials interpolation (UMMI) schemes are discussed and compared based on numerical tests and theoretical analysis.

RMMI interpolation scheme can be treated as an extension of the traditional SIMP/RAMP interpolation model reserved for the case of only one solid material phase and the void to the case of multiphase materials. This scheme is constructed through a recursive process. For a structure with $m$ solid materials and $n$ designable finite elements, the RMMI scheme interpolates Young's modulus of element $i$ in the following manner

$$
E_{i}=E\left(\mathbf{x}_{i}\right)=v_{i m}\left(x_{i m}\right) E_{i}^{(1, m)},
$$

where

$$
\begin{gathered}
E_{i}^{(m, m)}=E^{(m)}, \\
E_{i}^{(m-\xi, m)}=v_{i \xi}\left(x_{i \xi}\right) E_{i}^{(m-\xi+1, m)}+\left[1-v_{i \xi}\left(x_{i \xi}\right)\right] \\
E_{i}^{(m-\xi)} ;(\xi=1,2, \ldots, m-1), \mathbf{x}_{i} \\
=\left\{x_{i j}\right\} ;(i=1,2, \ldots, n ; j=1,2, \ldots, m),
\end{gathered}
$$

where $E_{(j)}$ denotes Young's modulus of material phase $j . \boldsymbol{x}_{i}$ denotes the set of design variables associated with element $i$. Clearly, each element has $m$ design variables that have different significations. $v_{i j}\left(x_{i j}\right)$ is the weighting function related to element $i$.

It is indicated that the nonlinearity of the mass constraint introduced by RMMI brings numerical difficulties to attain the global optimum of the optimization problem. On the contrary, the UMMI-2 scheme makes it possible to formulate the mass constraint in a linear form with separable design variables. 


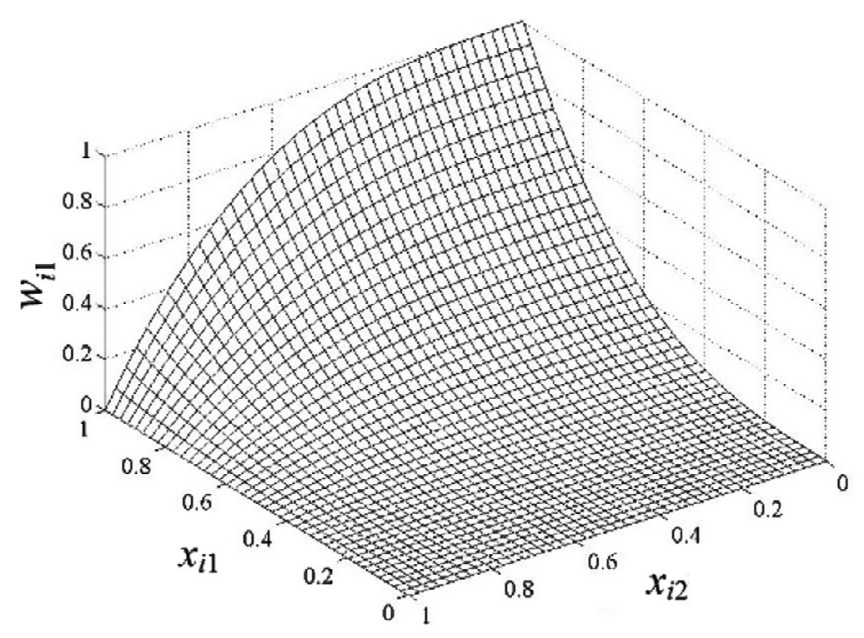

(a) $w_{i 1}$

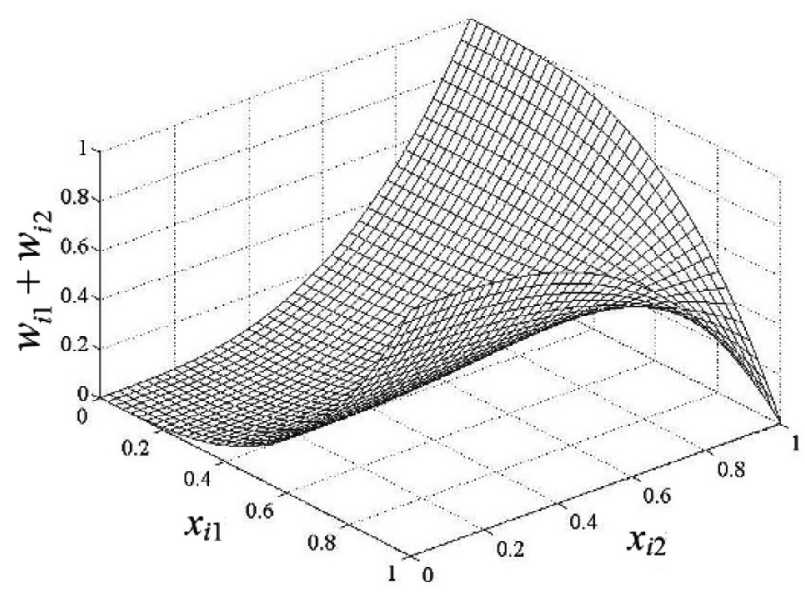

(b) $w_{i 1}+w_{i 2}$

Figure 1. UMMI-2 interpolation model with two solid materials $(m=2, p=3)$.

UMMI originated from the so-called DMO. It means that the continuous laminate design of fiber orientations was treated as a DMO problem. In this paper, the concept of DMO is generalized for structural topology optimization with multiphase materials. As the weighting function is uniform for each material phase, the interpolation scheme is, in fact, a uniform multiphase materials interpolation (UMMI). For a problem of $m$ solid materials and $n$ designable elements, each element will have $m$ design variables. The UMMI interpolation of Young's modulus can be expressed as the weighted sum of all candidate material phases:

$$
E_{i}=\sum_{j=1}^{m} w_{i j} E^{(j)}
$$

$w_{i j}$ is the weighting function associated with the $j$ th material phase which can be defined in UMMI-2 as

$$
\begin{gathered}
w_{i j}=x_{i j}^{p} \prod_{\xi=1}^{m}\left(1-x_{i \xi}^{p}\right) . \\
\xi \neq j
\end{gathered}
$$

As shown in Figure 1, one such formulation favors very much the problem resolution by means of mathematical programming approaches, especially the convex programming methods. Moreover, numerical analysis indicates that fully uniform initial weighting is beneficial to seek the global optimum when UMMI-2 scheme is used. Besides, the relationship between the volume constraint and mass constraint is theoretically revealed. The filtering technique is adapted to avoid the checkerboard pattern related to the problem with multiphase materials.

Numerical examples show that the UMMI-2 scheme with fully uniform initial weighting is reliable and efficient to deal with the structural topology optimization with multiphase materials and mass constraint. Meanwhile, the mass constraint formulation is evidently more significant than the volume constraint formulation.

As shown in Figures $2 a$ and $2 b$, with the RMMI scheme and uniform $x_{i j}$ or $w_{i j}$, the optimal structures have configurations consisting of both VM1 and VM2. Comparatively, the result shown in Figure 2c with only one single solid material (VM2) is found to be stiffer. This implies that the RMMI scheme cannot yield the global optimum at least even uniform initial weighting are used.

Using the UMMI-2 scheme with fully uniform initial variables, the optimal structure only consists of VM2, as shown in Figure 2d, even VM1 andVM2 are available. Although configurations given in Figures $2 \mathrm{c}$ and $2 \mathrm{~d}$ are very similar, the structural compliances using UMMI-2 are smaller. Besides, partial uniform initial variables of Case $\mathrm{B}$ are adopted and the results are shown in Figures 2e and 2f. Obviously, different initial variables lead to totally different configurations. Note that some elements at the right-bottom corner in Figure $2 \mathrm{f}$ consist of "mixed" materials.

Another interesting thing is to compare the optimization results obtained with mass and volume constraints. To do this, suppose the structure mass is less than $25.92 \times 10^{3} \mathrm{~kg}$. The upper bound of the volume constraint can be correspondingly determined for VM3 if the upper bound of the volume constraint of VM1 is fixed a priori. For different volume fractions of VM1, optimization results are plotted in Figure 3. The optimal solution with the mass constraint corresponds to the volume fractions of VM1 and VM3 being 0.463 and 0.068 , respectively. It can be seen that this is the best solution among all possible ones using volume constraint in the sense of compliance minimization. In other words, the mass constraint always leads to a better solution than the volume constraint with the precondition of the same structure mass. Meanwhile, the percentage of each solid material phase will be automatically identified. In fact, the mass constraint has a more significant and important sense than the volume constraint in most engineering problems. 


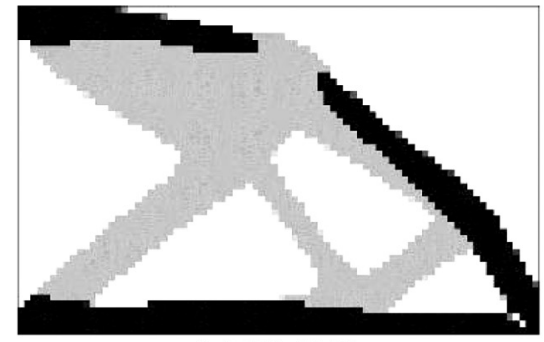

(a) RMMI

VM1-VM2, uniform $x_{i j}$

$\mathrm{C}=172.58$

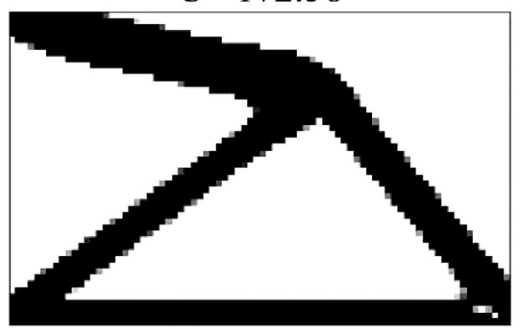

(d) UMMI-2

$\mathrm{VM} 1-\mathrm{VM} 2, x_{i 1}=x_{i 2}=0.2$

$\mathrm{C}=157.03$

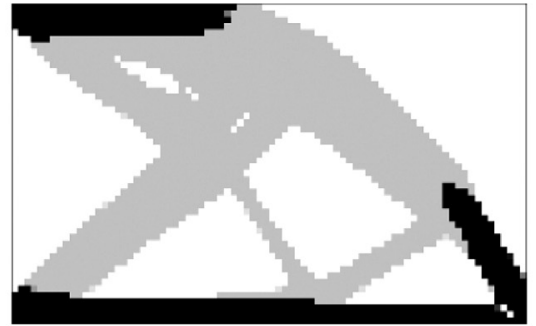

(b) RMMI

VM1-VM2, uniform $w_{i j}$

$\mathrm{C}=179.17$

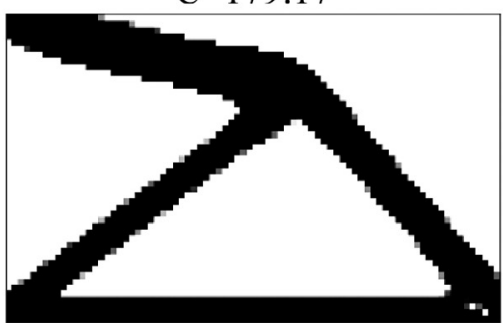

(e) UMMI-2

VM1-VM2, $x_{i 1}=0.1 x_{i 2}=0.4$

$\mathrm{C}=156.60$

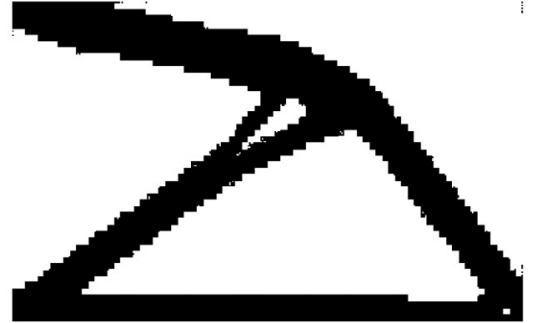

(c) RMMI

VM2

$\mathrm{C}=159.59$

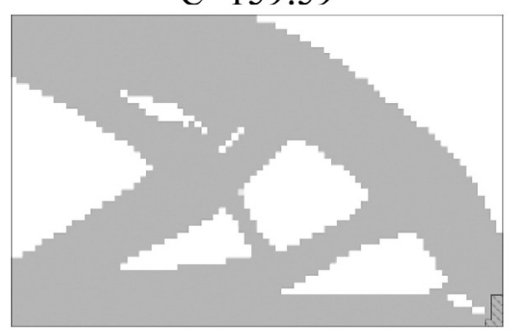

(f) UMMI-2

VM1-VM2, $x_{i 1}=0.4 x_{i 2}=0.1$

$\mathrm{C}=286.65$

Figure 2. Optimization results of a cantilever beam.

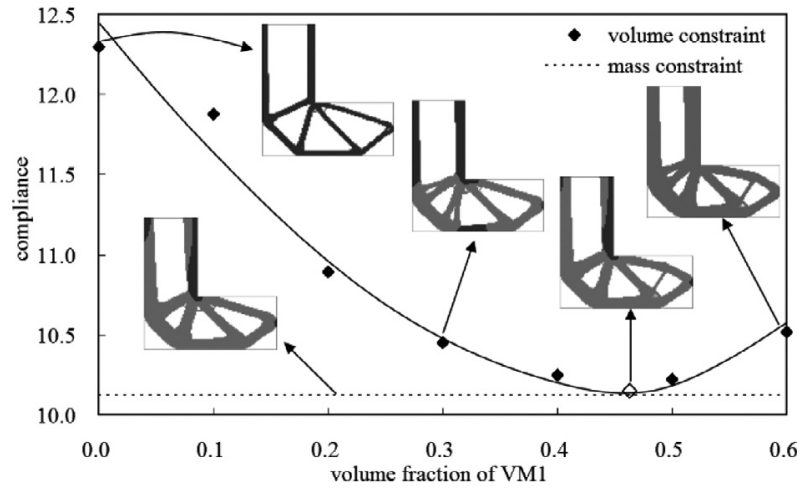

Figure 3. Comparisons of the optimization results under the mass and volume constraints with the equal structure mass.

From the development of this work, it is seen that the combination of UMMI-2 scheme together with fully uniform initial variables and the convex programming methods provides an efficient approach for the structural layout design of multiphase materials in static load case when the mass is controlled. Meanwhile, due to its generality, this approach may be extended to the structural design and microstructure design of multiphase materials in generalized load cases.

\section{Integrated layout and topology optimization}

In many engineering products, typical structural systems consists of components and structures with integrated interconnections inside a limited packing space. Global system performances significantly depend upon the layout of components and the topology of supporting structures. The design of multicomponent structural systems is a relatively new area and is still not extensively researched. Since most real life engineering design problems involve multiple components or structures, it is a subject of great relevance

Recently, further researches are carried out in our group with significant improvements and systematical methodologies $[3,4]$. Packing optimization is performed by means of the FCM (Finite Circle Method), and the supporting structure is designed by means of topology optimization techniques. Because movable components inevitably change the FE mesh and locally corrupt the updating of the material distribution, the traditional topology optimization method using a fixed FE mesh is no longer applicable. To circumvent this problem, a density point technique was proposed to associate the density variables with the centroids of a fixed background mesh. As shown in Figure 4, density points are a set of predefined points in the design domain to control the material distribution. No matter how the mesh changes, each element receives the pseudo-density value from its nearest density point.

In order to reduce the computing cost and simplify sensitivity analysis with respect to location design variables of each component, the super-element technique is adopted to model the movable components.

Similar to classic topology optimization, the sensitivity of $C$ with respect to pseudo-density design variables can be analytically obtained by

$$
\frac{\partial C}{\partial \eta_{i}}=\frac{\partial \mathbf{G}^{T}}{\partial \eta_{i}} \cdot \mathbf{u}-\frac{1}{2} \mathbf{u}^{T} \cdot \frac{\partial \mathbf{K}}{\partial \eta_{i}} \cdot \mathbf{u} .
$$



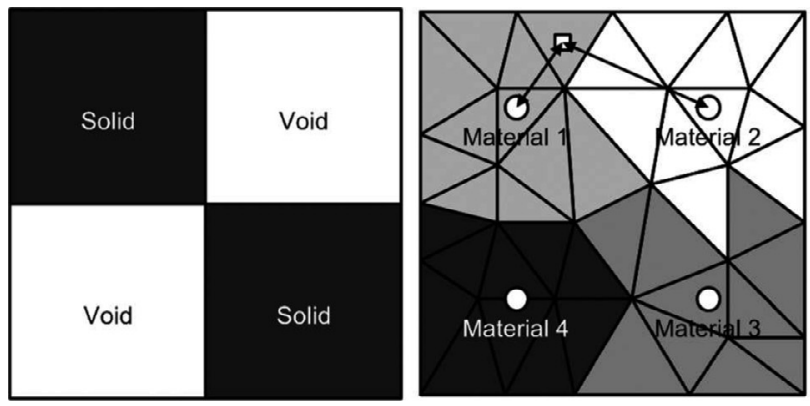

Density points

$\square$ Centroid of element

Figure 4. Element material properties received from the density points.

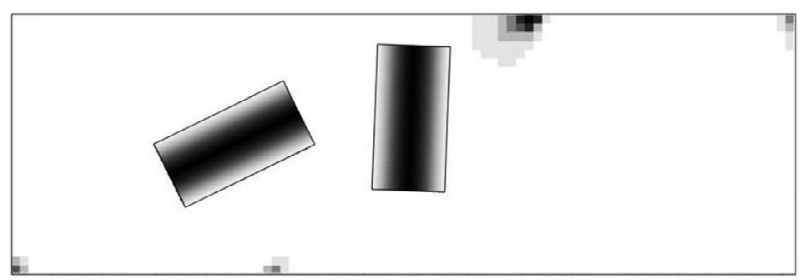

(a)

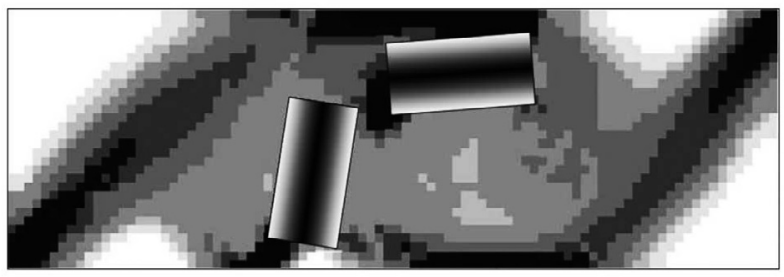

(b)

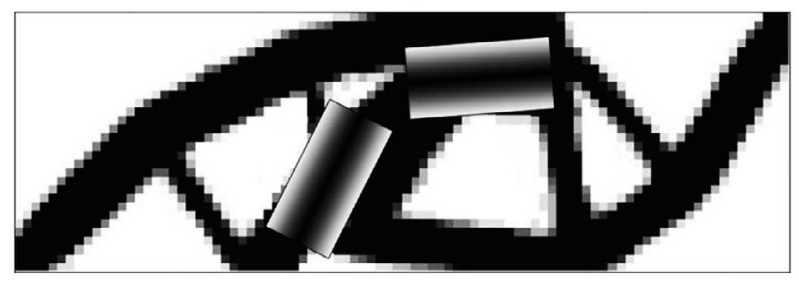

(c)

Figure 5. Integrated layout design with components of stiffer materials [40]: (a) iteration 5, (b) iteration 20, and (c) final design; final strain energy C50.074 J.

As to the sensitivity with respect to location design variables of components, a semi-analytical scheme combined with super-element formulation is applied in the following way. The global stiffness matrix of the system is partitioned into three parts: supporting structure, mesh transition region, and components. The derivative of the system compliance with respect to $s_{\varepsilon}$ representing one of the three location parameters $x_{\varepsilon}, y_{\Theta}$ and $\theta_{\varepsilon}$ of component $\varepsilon$ gives rise to

$$
\frac{\partial C}{\partial \boldsymbol{s}_{\varepsilon}}=\mathbf{u}^{T}\left[\frac{\partial \mathbf{G}^{T}}{\partial \boldsymbol{s}_{\varepsilon}}-\frac{1}{2}\left(\frac{\partial \mathbf{K}_{(S)}}{\partial \boldsymbol{s}_{\varepsilon}}+\sum_{i=1}^{n_{c}} \frac{\partial \mathbf{K}_{(T) l}}{\partial \boldsymbol{s}_{\varepsilon}}+\sum_{i=1}^{n_{c}} \frac{\partial \mathbf{K}_{(C) l}}{\partial \boldsymbol{s}_{\varepsilon}}\right) \mathbf{u}\right] .
$$

A design example of simple structure system with two rectangular components is considered here. The global strain energy is the objective function to be minimized and the volume fraction is set to be $50 \%$. Assume that all components are made of steel, and the supporting structure is made of aluminum. As shown in Figure 5, the components and the supporting structure are optimized simultaneously. Finally, the components are located in the main loading path of the structure due to their high stiffness.

Design of multicomponent systems can also be extended to support layout of bridge-like problems. Fixations are, here, defined as movable solid support components, which are partially embedded into the design domain. Positions of these support components are optimized together with the structural topology to obtain the maximum stiffness of the global system.

A typical example is illustrated in Figure 6. Materials used for the structure and support components are aluminum and steel, respectively. The road on the bridge is a non-designable area. Four identical support components are located symmetrically along the bottom boundary of the design domain. A uniform surface pressure is applied on the top of the bridge. The volume fraction for the design domain is set to be $40 \%$. By optimization, it is found that a slight change of the support locations will lead to a significant variation of the structural topology.

It is possible to extend the methodology to the layout design of 3D multicomponent systems. However, the embedded meshing technique for 3D problems is much more complicated than in $2 \mathrm{D}$ situation. Meanwhile, the semi-analytical sensitivity analysis scheme for location design variables of components is time-consuming because each 3D component has now six location design variables. Recently, a Heaviside function-based approach is investigated for sensitivity analysis with respect to location design variables. The material discontinuity across the component boundary is approximately smoothed by means of the Heaviside function. When a location design variable is perturbed, sensitive elements attached to the component boundary are assumed to undertake a material property change, while the mesh remains unchanged. In this way, sensitivity analysis with respect to location design variables is carried out as easily as for pseudo-density variables. Figure 7 shows the optimization result of a $3 \mathrm{D}$ structure, in which tetrahedral elements are used and the involved components have a greater Young's modulus than that of the supporting structure. 


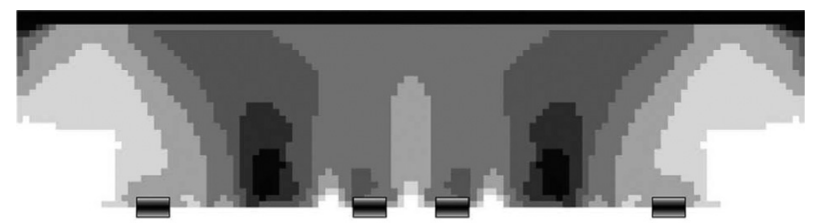

(a)

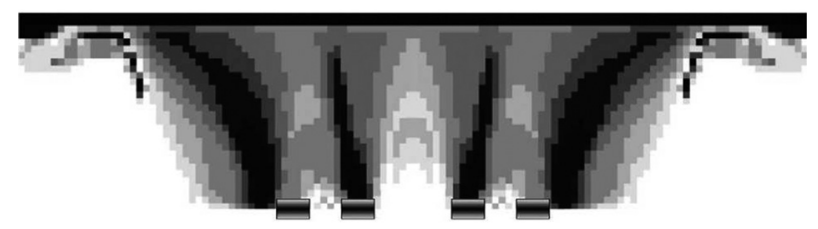

(b)

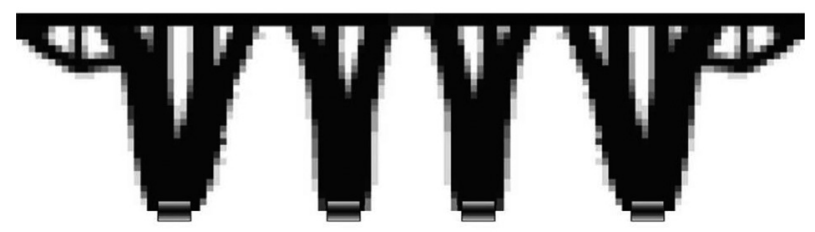

(c)

Figure 6. Integrated support and layout design of a bridge-like structure: (a) iteration 5, (b) iteration 10, and (c) final design, C50.1298 J.

\section{Prediction of effective material properties}

This work presents a microstructural modeling method considering the oxidation effect. Further studies on the prediction of material properties were carried for the multiphase and multilayer braided composite [5]. Regarding the multi-scale characteristics of the composite, the microstructure modeling is carried out sequentially from fiber to tow scale. The geometrical configuration of the microstructure is first analyzed, and mathematical relations among different geometrical parameters are derived on each scale. Second, effective elastic properties are obtained based on the sequential homogenization from fiber to tow scale. A strain energy based method is proposed to evaluate effective elastic properties with specific boundary conditions imposed on the microstructure. Numerical results obtained by the proposed method and the microstructure model show a good agreement with the results measured experimentally.

The present study aimed at the microstructure modeling and numerical prediction of elastic properties of 3D four-step MPML braided composites. First, the microstructure modeling is carried out sequentially from fiber to tow scale. Geometrical descriptions and finite element modeling of the microstructure are presented in detail.

The RVC (Representative Volume Cell) model of the 3D four-step MPML braided composite involves two scales: fiber and tow scales. The first scale concerns the modeling of RVC for MPML tows, and the second scale concerns the modeling of RVC for 3D four-step MPML braided composites as shown in Figure 8. Considering the multi-scale characteristics, the multi-scale analysis methods are needed for the analysis of MPML plain weave composites. The multi-scale analysis procedure is as follows. First, on the fiber scale, finite element model is built to obtain the effective moduli of the fiber scale $\mathrm{RVC}$, and the results are used for the tows that were treated as a homogeneous transversely isotropic material. Second, based on the obtained elastic properties of tows, finite element model of the tow scale RVC is created to evaluate effective elastic properties of the composite. Here, RVC models of both scales are established using the ANSYS finite element software.

On the tow scale, the finite element model of RVC is depicted in Figure 9. Here, a 10-node tetrahedral solid element SOLID187 is used for the discretization, and the finite element models of both tows and matrices are shown respectively.

Second, a strain energy based method is applied to evaluate the effective elastic properties. Numerical results are then compared with the experimental results to validate the modeling.

In the elastic regime, the macroscopic behaviors of the RVC can be characterized by the effective stress tensor $\boldsymbol{\sigma}$ and strain tensor $\boldsymbol{\varepsilon}$ over the homogeneous equivalent model. They are interrelated by the effective, also termed homogenized, stiffness matrix $\boldsymbol{C}^{H}$

$$
\sigma=\mathbf{C}^{H} \varepsilon
$$

The matrix coefficient $\mathbf{C}^{H}{ }_{111}$ can be derived

$$
\mathbf{C}_{1111}^{H}=2 E^{(1)} / V \text {. }
$$

In the same way, demonstrations can be made for other coefficients. The effective stiffness matrix can be written as

$$
\left[\begin{array}{cccccc}
\frac{2 E^{(1)}}{V} & \frac{E^{(7)}-E^{(2)}-E^{(1)}}{V} & \frac{E^{(9)}-E^{(3)}-E^{(1)}}{V} & 0 & 0 & 0 \\
\frac{2 E^{(2)}}{V} & \frac{E^{(8)}-E^{(3)}-E^{(2)}}{V} & 0 & 0 & 0 \\
& \frac{2 E^{(3)}}{V} & 0 & 0 & 0 \\
& & \frac{2 E^{(4)}}{V} & & \\
& & & \frac{2 E^{(5)}}{V} & \\
& & & & \frac{2 E^{(6)}}{V}
\end{array}\right] .
$$

The elastic constants can be derived by inversing the above matrix. In practice, the considered RVC will be discretized as a finite element model on which the initial strain will be imposed to evaluate the strain energy. The comparison with the experimental results shows the validity and the rationality of the present method. Future studies will be focused upon the numerical prediction of thermal properties (the thermal expansion coefficient and thermal conductivity), progressive failure analysis and design optimization of the microstructure for 3D MPML braided composite.

Similar model incorporates the modeling of oxidized microstructure and computing of degraded elastic moduli can also be extended to simulate the oxidation behaviors of $2 \mathrm{D} \mathrm{C} / \mathrm{SiC}$ composites exposed to air oxidizing environments below $800{ }^{\circ} \mathrm{C}$ 


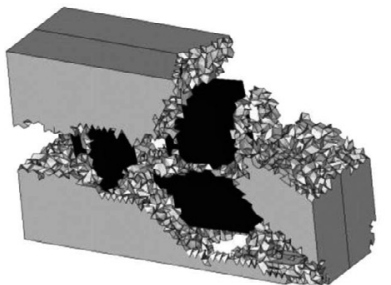

(a)

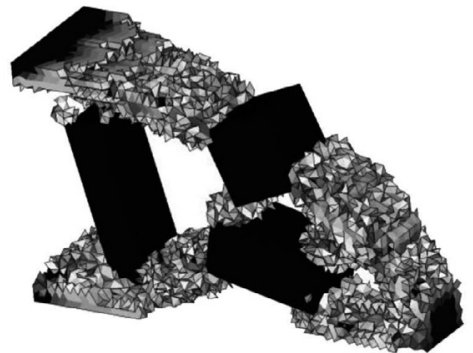

(d)

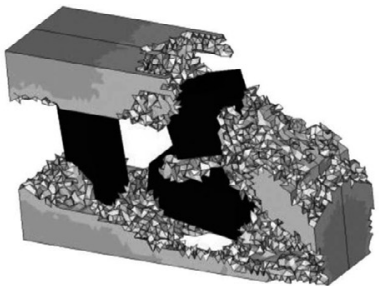

(b)

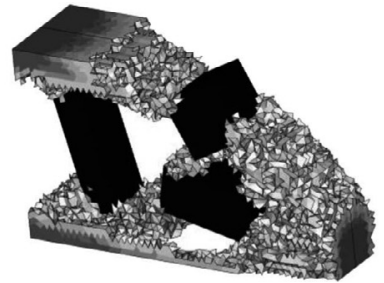

(c)

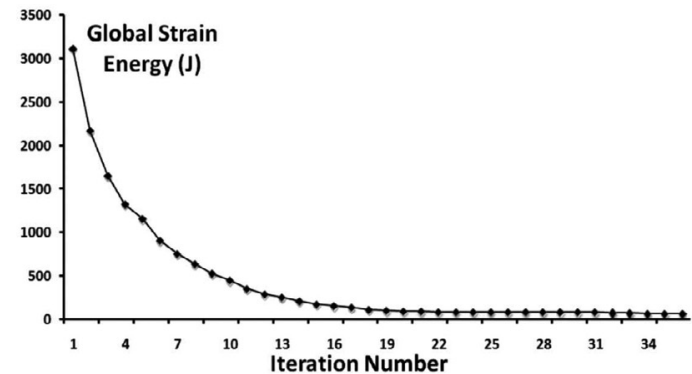

(e)

Figure 7. Integrated layout design of a 3D structure with three components: (a) iteration 2, (b) iteration 5, (c) iteration 10, (d) iteration 18, and (e) iteration history.

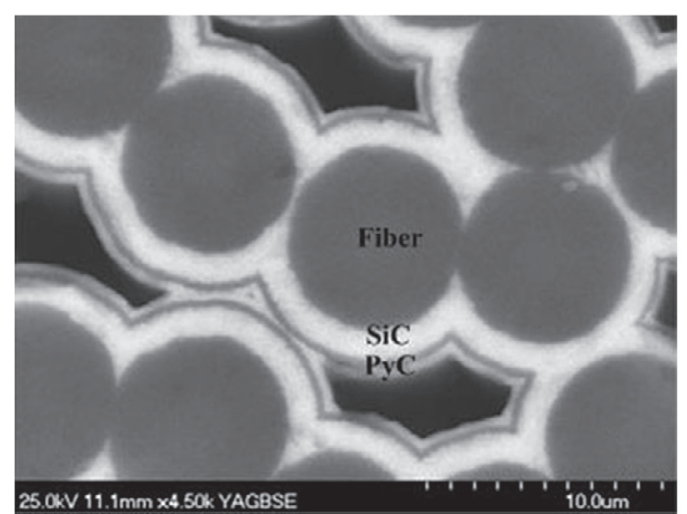

(a) fiber scale

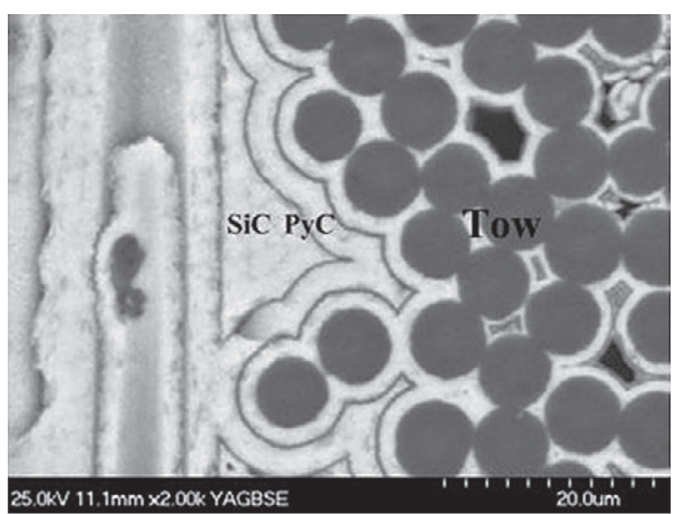

(b) tow scale

Figure 8. Images (SEM) of 3D braided MPML C/C-SiC.

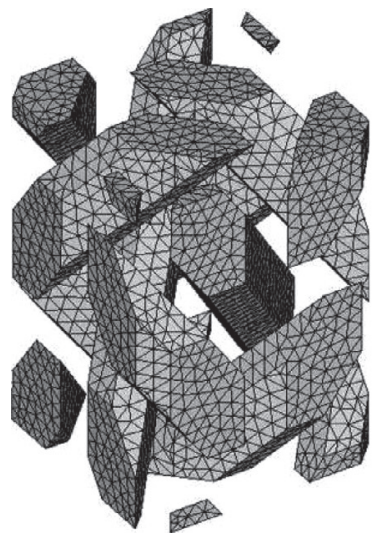

(a)

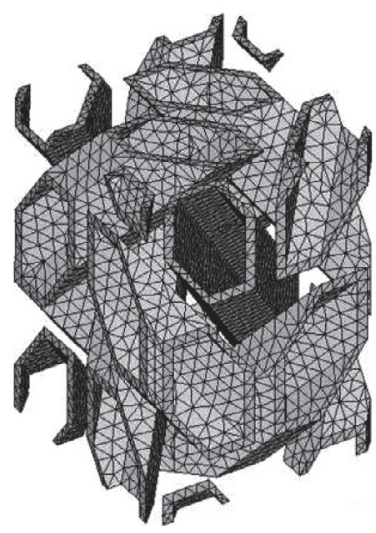

(b)

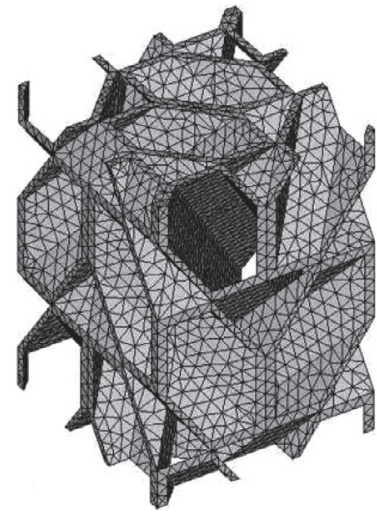

(c)

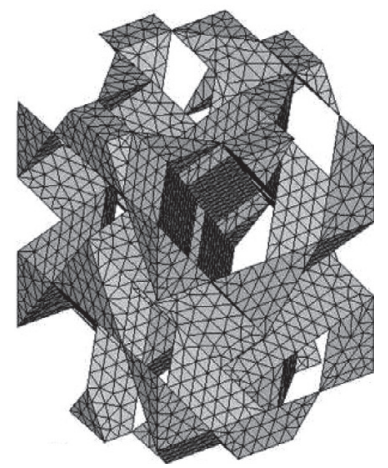

(d)

$\begin{array}{llll}\text { (a) Tow } & \text { (b) First layer of matrix } & \text { (c) Second layer of matrix } & \text { (d) Last layer of matrix }\end{array}$

Figure 9. Finite element model of RVC on tow scale. (a) Tow, (b) First layer of matrix, (c) Second layer of matrix, and (d) Last layer of matrix. 

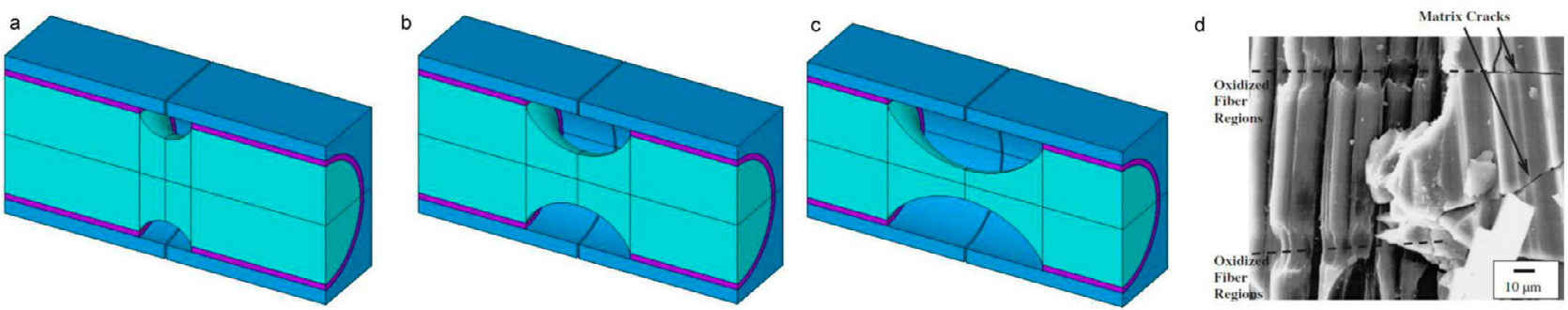

(a) $10 \mathrm{~h} \mathrm{(b)} 20 \mathrm{~h}$ (c) $30 \mathrm{~h}$ and (d) experimental observation

Figure 10. Morphologies of microscopic RVC at different oxidation time. (a) 10 h, (b) 20 h, (c) 30 h, and (d) experimental observation.

[6]. Regarding the multi-scale characteristics of $2 \mathrm{D} \mathrm{C} / \mathrm{SiC}$ composite, the microstructure modeling is carried out on microscopic and macroscopic scale, respectively to compute the degraded elastic properties in terms of time duration, temperature and pressure, whose influences upon the oxidation microstructure morphology and degraded properties of $2 \mathrm{D} \mathrm{C} / \mathrm{SiC}$ composites are also investigated. The predicted microstructure morphology after oxidation is found in good agreement with experiment data as shown in Figure 10.

It is found that the computed in-plane extensional moduli after oxidation are in good agreement with experimental data over the oxidation time. In addition, numerical results show that the temperature and pressure have a profound influence upon the oxidation results. The ablation of carbon phases is aggravated with the increase of temperature and pressure and therefore the elastic modulus of composite decreases gradually. An immediate perspective is to address other composite architectures such as the $3 \mathrm{D}$ braided $\mathrm{C} / \mathrm{SiC}$ composite and to extend the developed oxidation model to other oxidizing environments, like water or gas-water environments. Besides, it is worth to note that the oxidation simulation of $2 \mathrm{D} \mathrm{C} / \mathrm{SiC}$ composite above $800{ }^{\circ} \mathrm{C}$ is not taken into account in the current work because the oxidation scheme is non-uniform and much complicated.

\section{Composite design}

Composite design with fiber orientation optimization plays an important role in aircraft structure design. Earlier orientation optimization methods face the main problem of huge number of design variables. Recently, a patch concept was proposed to reduce the number of design variables as shown in Figure 11. The elements in one patch share identical definition of fiber orientation. However, the traditional stress-based method cannot deal with patch orientation optimization of composite structures. In this paper, we propose an extended stress-based method to deal with such problems. The considered problems are to minimize the mean compliance under multiple load cases or to maximize the eigenvalues of a composite structure. Four numerical examples are solved in order to demonstrate the efficiency of the new method. It is shown that the new method has the ability to deal with constraints on orientation angle, such as symmetric, anti-symmetric and discrete orientation angle constraints. The iteration is less time-consuming because no sensitivity analysis is needed and a quick convergence rate can be achieved.

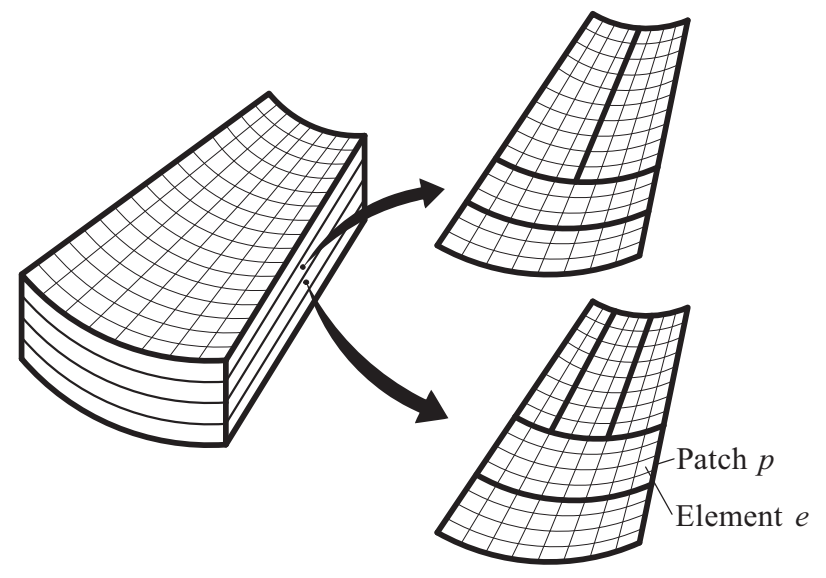

Figure 11. Definitions of layers, patches, and finite elements.

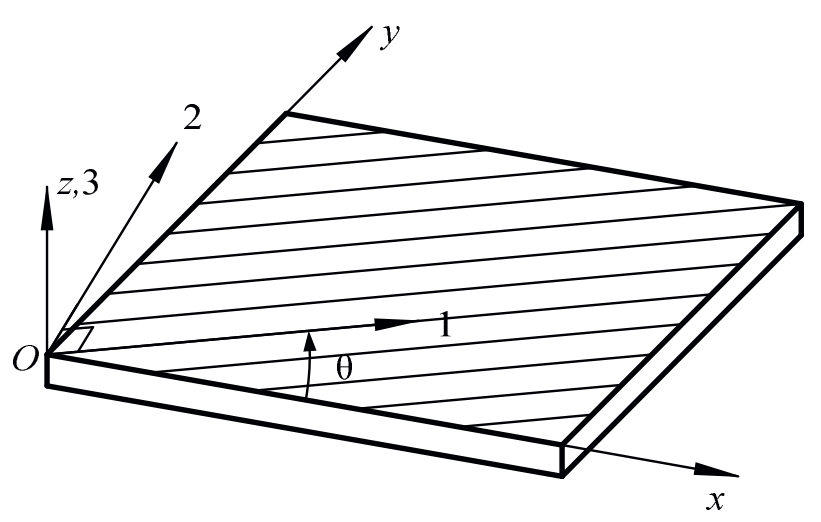

Figure 12. Orthotropic material.

In this work [7], the stress-based method is extended to deal with optimal patch orientation problems for composite structures under single and multiple load conditions. Some special constraints related to patch orientation angles are applied for the symmetric and anti-symmetric laminates in which patch orientation angles have to take the same and the opposite values, respectively. Besides, according to manufacturing considerations, investigations are made to the cases where the 


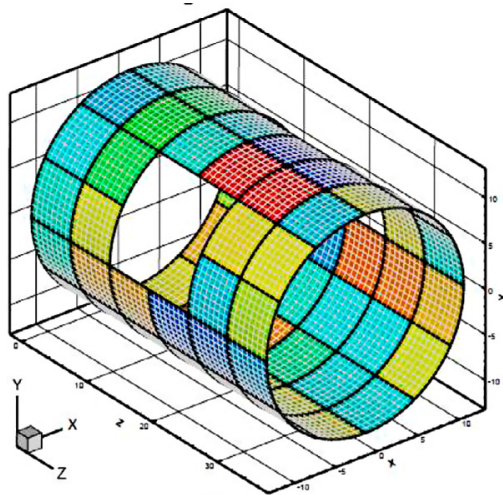

Layer 1

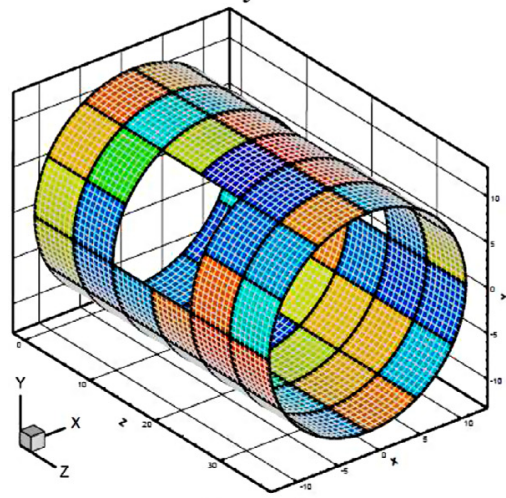

Layer 3

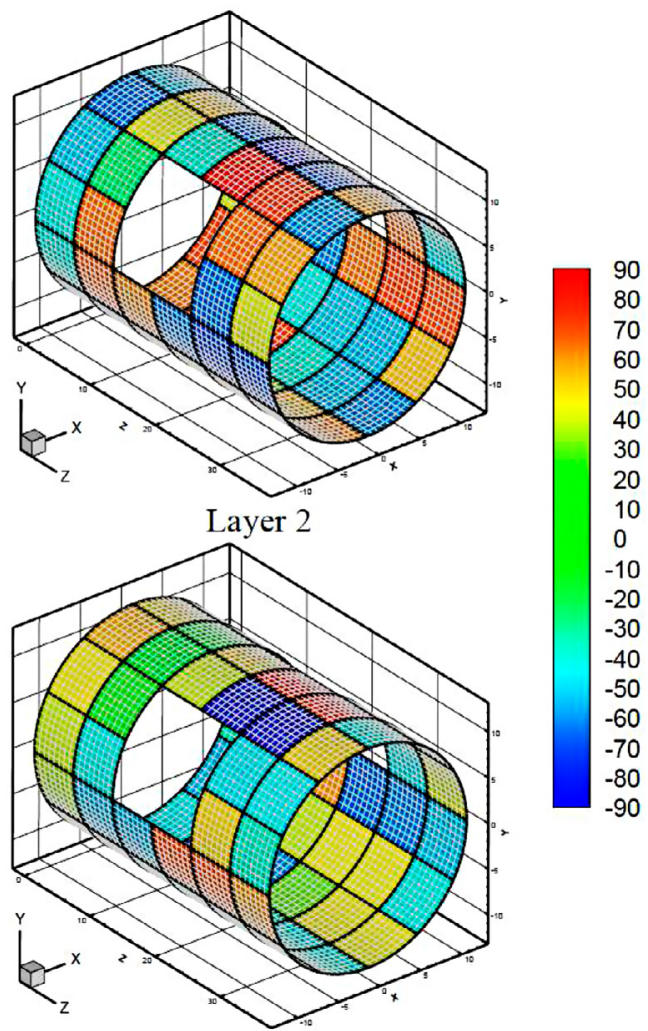

Layer 4

Figure 13. Optimal patch orientation angles with anti-symmetric laminate.

orientation angles are restricted to discrete values of $0^{\circ}, 45^{\circ}$, $-45^{\circ}$, and $90^{\circ}$. Several examples are finally given to show the validity of this method.

For thin laminated composite structures, the plane-stress condition may be applied due to the fact that out-of-plane stresses are often small compared with in-plane-stresses. Suppose each layer of the laminate is made of orthotropic materials. Figure 12 shows a patch over a layer where the stress-strain relationship of a point is

$$
\left\{\begin{array}{c}
\varepsilon_{1} \\
\varepsilon_{2} \\
\gamma_{12}
\end{array}\right\}=\left[\begin{array}{ccc}
S_{11} & S_{12} & 0 \\
S_{12} & S_{22} & 0 \\
0 & 0 & S_{66}
\end{array}\right]\left\{\begin{array}{c}
\sigma_{1} \\
\sigma_{2} \\
\tau_{12}
\end{array}\right\}=\mathrm{S}_{0}\left\{\begin{array}{c}
\sigma_{1} \\
\sigma_{2} \\
\tau_{12}
\end{array}\right\}
$$

where $\boldsymbol{\varepsilon}=\left\{\varepsilon_{1}, \varepsilon_{2}, \gamma_{12}\right\}^{T}$ and $\boldsymbol{\sigma}=\left\{\sigma_{1}, \sigma_{2}, \tau_{12}\right\}^{\mathrm{T}}$ represent the planar strains and stresses in the primed coordinate system $(O-123)$, respectively; $\boldsymbol{S}_{0}$ is the un-rotated orthotropic compliance matrix.

Unlike the traditional stress-based method related to each element, the Extended stress-based method (ESBM) expands the integration over the patch domain so that

$$
\frac{\partial \Phi}{\partial \theta_{p}}=\int_{\Omega_{p}} \sigma^{T} \frac{\partial \mathrm{S}}{\partial \theta_{p}} \sigma \mathrm{d} \Omega=0
$$

where $\theta_{p}$ is the orientation variable related to patch $p$; $\Omega_{p}$ represents the spatial domain of patch $p$.
Let $\sigma_{x}, \sigma_{y}$ and $\tau_{x y}$ denote components of the stress tensor in the unprimed coordinate system $(O-x y z)$, equation (12) can explicitly be developed as a function of the patch orientation variable $\theta_{p}$

$$
A \sin 2 \theta_{p}+B \cos 2 \theta_{p}+D \sin 4 \theta_{p}+E \cos 4 \theta_{p}=0 .
$$

where coefficients $A, B, D$ and $E$ are computed as

$$
\begin{gathered}
A=c \int_{\Omega_{p}}\left(\sigma_{x}^{2}-\sigma_{y}^{2}\right) \mathrm{d} \Omega, \\
B=2 c \int_{\Omega_{p}} \tau_{x y}\left(\sigma_{x}+\sigma_{y}\right) \mathrm{d} \Omega, \\
D=d \int_{\Omega_{p}}\left[4\left(\tau_{x y}\right)^{2}-\left(\sigma_{x}-\sigma_{y}\right)^{2}\right] \mathrm{d} \Omega, \\
E=4 d \int_{\Omega_{p}} \tau_{x y}\left(\sigma_{x}-\sigma_{y}\right) \mathrm{d} \Omega .
\end{gathered}
$$

Note that the numerical Gaussian integration can be applied for computing coefficients in equation (13). In practice, if the finite element mesh is dense enough, the stress in each element can be assumed to be a constant approximated by the value at the centroid of the element.

When $\theta_{p} \neq \pi / 2$, roots of equation (13) can equivalently be obtained by solving the following fourth-order polynomial equation in terms of $\tan \theta_{p}$ within the interval $(-\pi / 2, \pi / 2)$.

$$
\begin{array}{r}
(E-B) \tan ^{4} \theta_{p}+(2 A-4 D) \tan ^{3} \theta_{p}-6 E \tan ^{2} \theta_{p} \\
+(2 A+4 E) \tan \theta_{p}+B+E=0
\end{array}
$$


By means of equation (13), the following evaluation function can be defined to determine the optimal value of $\theta_{p}$ selected from the real roots of equation (15) and $\theta_{p}=\pi / 2$.

$$
\begin{aligned}
& f\left(\theta_{p}\right)=\int \frac{\partial \Phi}{\partial \theta_{p}} \mathrm{~d} \theta_{p} \\
& =\int\left(A \sin 2 \theta_{p}+B \cos 2 \theta_{p}+D \sin 4 \theta_{p}+E \cos 4 \theta_{p}\right) \mathrm{d} \theta_{p} \\
& =\frac{1}{4}\left(-2 A \cos 2 \theta_{p}+2 B \sin 2 \theta_{p}-D \cos 4 \theta_{p}+E \sin 4 \theta_{p}\right) \\
& \quad+\text { constant. }
\end{aligned}
$$

Note that if each patch has just one finite element, the ESBM is identical to the traditional stress-based method.

One example as shown in Figure 13 is to show the validity of the ESBM in dealing with constraints of patch orientation variables. The number of patches is 64 and each patch consists of $8 \times 8$ elements. The left side of the cylinder $(Z=0)$ is clamped and a bending moment $M x$ is applied on the right side $(Z=36)$ of the structure. Assume the laminated composite with four layers is used and they are numbered sequentially from the inside to the outside of the cylinder. Likewise, the objective function is to minimize the structural mean compliance.

Acknowledgements. This work is supported by National Natural Science Foundation for Distinguished Young Scholar (10925212), National Natural Science Foundation of China (90916027, 11002113, 51275424, 11172236), 973 Program (2011CB610304), the 111 Project (B07050), NPU Foundation for Fundamental Research (JC20120229).

\section{References}

1. Gao T, Zhang WH. 2011. A mass constraint formulation for structural topology optimization with multiphase materials. International Journal for Numerical Methods in Engineering, 88(8), 774-796.

2. Gao T, Zhang WH, Duysinx P. 2011. Topology optimization of structures designed with multiphase materials: volume constraint or mass constraint? Chinese Journal of Theoretical and Applied Mechanics, 43(2), 296-305.

3. Zhang WH, Xia L, Zhu JH, Zhang Q. 2011. Some recent advances in the integrated layout design of multicomponent systems. Journal of Mechanical Design, 133(10), 104503-1-15.

4. Xia L, Zhu JH, Zhang WH. 2011. New strategies for the efficient integrated layout design of multi-component system. Journal of Mechanical Engineering, 46(10), 148-155.

5. Xu YJ, Zhang WH, Domaszewski M. 2011. Microstructure modelling and prediction of effective elastic properties of $3 \mathrm{D}$ multiphase and multilayer braided composite. Material Science and Technology-London, 27(7), 1213-1221.

6. Xu YJ, Zhang WH. 2011. Numerical modelling of oxidized microstructure and degraded properties of $2 \mathrm{D} \mathrm{C} / \mathrm{SiC}$ composites in air oxidizing environments below 800 degrees C. Material Science and Engineering A, 528(27), 7974-7982.

7. Zhang J, Zhang WH, Zhu JH. 2011. An extended stress-based method for orientation angle optimization of laminated composite structures. Acta Mechanica Sinica, 27(6), 977-985.

Cite this article as: Zhu JH \& Zhang WH: Structural optimization in ESAC: annals 2011. Int. J. Simul. Multisci. Des. Optim., 2014, 5, A09. 